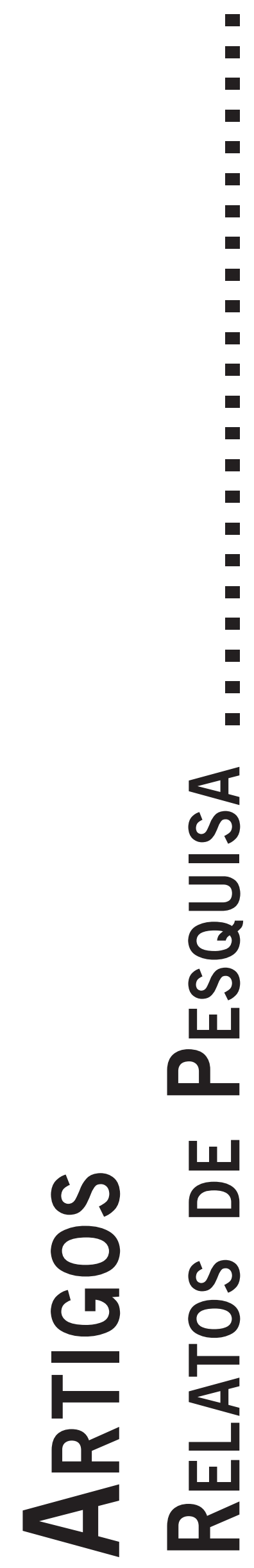




\title{
PROPOSTA DE ESTÁGIO SUPERVISIONADO PARA ATUAÇÃO DE PSICÓLOGOS NA SAÚDE PÚBLICA
}

\author{
Training proposal to the psychologist's professional practice in Public Health
}

Propuesta de prácticas supervisadas para psicólogos en la Sanidad Pública

Railda Fernandes Alves

Emily SouZa Gaiño

Gabriella CÉZar dos SANTos

Luisa de Marillac Ramos Soares

\begin{abstract}
Resumo: Neste artigo discutimos a formação profissional e suas lacunas para a atuação do psicólogo na saúde pública que não oferece ferramentas teóricas, técnicas e críticas para o trabalho no SUS. Problematizamos o privilégio dado ao enfoque clínico tradicional e apresentamos uma proposta de estágio supervisionado fundamentada na Psicologia da Saúde, na Abordagem Centrada na Pessoa e na Política Nacional de Humanização. Sugerimos um rol de intervenções organizadas a partir da lógica dos níveis primário, secundário e terciário de saúde, que vão da psicoterapia às ações multiprofissionais até a mediação pedagógica da equipe multiprofissional de saúde.
\end{abstract}

Palavras-chave: Psicologia; Saúde pública; Estágio clínico; Capacitação profissional.

Abstract: On this article, we discuss the professional training and its gaps for the psychologist's professional practice on public health, which doesn't offer theoretical, technical and critical tools to SUS (Unified Health System)'s work. We problematize the privilege given to the traditional clinical focus and we presented a proposal of supervised training based on the Health's Psychology, Person-Centered Approach and National Humanization Policy. We suggest an intervention list organized from the health's primary, secondary and tertiary levels, ranging from psychotherapy to multidisciplinary actions until the health multidisciplinary group's pedagogical mediation.

Keywords: Psychology; Public health; Clinical stage; Professional training.

Resumen: En este artículo discutimos la formación de los profesionales y sus insuficiencias en la actuación del psicólogo en la sanidad pública, al no ofrecer herramientas teóricas, técnicas y críticas para su labor en la Seguridad Social. Trabajamos la problemática del privilegio dado al enfoque clínico tradicional y presentamos una propuesta para la realización de las prácticas fundamentada en la Psicología de la Salud, en el Enfoque Centrado en la Persona y en la Política Nacional de Humanización. Proponemos un rol de intervenciones organizadas a partir de los niveles primario, secundario y terciario de salud, que va desde la psicoterapia a las acciones multiprofesionales y hasta la mediación pedagógica del equipo multiprofesional.

Palabras-clave: Psicología; Salud pública; Prácticas clínicas; Capacitación profesional.

\section{Introdução}

As mudanças no perfil epidemiológico das populações mundiais, que alteram a compreensão do que seja saúde-doença e morte, têm evidenciado a necessidade da criação de novas políticas e programas públicos de saúde. Tais políticas e programas, na medida em que são inaugurados, ratificam a importância das ações multiprofissionais no campo da saúde e apontam um aumento da demanda de psicólogos para atender as necessidades do campo.

Atualmente, no Brasil, as práticas dos psicólogos têm sido diversificadas e ampliadas para os novos setores públicos que figuram tanto na saúde através das políticas do Sistema Único de Saúde (SUS), como na Assistência Social, através das políticas do Sistema Único de Assistência Social (SUAS). Dados do
Censo SUAS/CREAS, desenvolvido pelo Ministério de Desenvolvimento Social e Combate à Fome [MDSCF] (2011), apresentaram que o profissional de Psicologia (18\%) está entre os três mais presentes nos serviços de políticas públicas de Assistência Social brasileira, o que justifica sua relevância nesse contexto. Os demais profissionais são os de nível médio (27,5\%), seguidos de Assistentes Sociais (21,5\%). Em relação aos programas do SUS há, segundo Macedo e Dimenstein (2011), de acordo com os registros do Cadastro Nacional de Estabelecimentos de saúde - CNES, 33.613 psicólogos em atuação no país. Esses mesmos autores enfatizam um significativo aumento da atuação dos profissionais de psicologia no âmbito da saúde pública brasileira entre os anos de 2006 e 2011, destacando que o número de psicólogos, nesse campo, quase triplicou nesse período. 
Para atender bem a essas novas configurações dos fazeres psicológicos no Setor da Saúde Pública, o psicólogo necessita receber uma formação (para os que ainda estão na Universidade) ou uma capacitação (para os que já estão na Rede de Saúde) capaz de mostrar-lhe a amplitude das ações em que pode atuar. A lógica dessa formação/ capacitação é preparar para uma atuação na saúde que seja compreendida e organizada de acordo com os níveis de atenção à saúde propostos pelo SUS.

Este argumento encontra fôlego na crítica feita aos Projetos Políticos Pedagógicos (PPP) dos Cursos de Psicologia que, apesar das exigências de transformação das práticas advindas do mercado de trabalho, ainda superestimam o modelo clínico tradicional cujo foco é a psicoterapia e a formação acadêmica para atuação na clínica privada.

A Psicologia tem em suas raízes o paradigma clínico, centrado no modelo médico (Figueiredo, 2010), modo de ver que influenciou os currículos acadêmicos (produtores do saber erudito), bem como as representações leigas (produzidas pelo senso comum) a fortalecerem a visão clínica da Psicologia. Bock, Furtado \& Teixeira (2002) afirmaram que no século XX, o Behaviorismo, a Psicologia da Gestalt e a Psicanálise foram as teorias psicológicas que mais se destacaram no cenário mundial, sendo o modelo clínico-individual predominantemente utilizado.

A clínica ${ }^{1}$, muitas vezes utilizada como sinônimo de psicoterapia, não se resume a ela. A psicoterapia deve ser compreendida como um saber localizado, específico da atenção especializada. Já a intervenção do psicólogo em saúde deve se ampliar para incluir outras práticas que deem conta da atenção à saúde mental dos indivíduos em todos os níveis, desde a atenção primária/básica até a alta complexidade. Na atualidade, a Psicologia em sua amplitude, diversidade e dinamismo teórico-prático avança em direção a novas modalidades de intervenção profissional. Como em outros campos do saber, a necessidade de novas práticas pressiona a produção de conhecimento acadêmico indicando a urgência em ratificar, em termos científicos, os novos fazeres que se configuram em função da transformação do campo de práticas em saúde.

Para auxiliar o debate de modernização dos saberes psicológicos e de ampliação das ações do psicólogo no campo multifacetado da saúde, apresentamos esta contribuição teórica, a qual se fundamenta em três argumentos: o primeiro focado na Psicologia da Saúde (PS), o segundo na Abordagem Centrada na Pessoa (ACP) e o terceiro

\footnotetext{
A Psicologia Clínica tradicional de acordo com Lo Bianco, Bastos \& Silva (1994) é centrada no enfoque intrapsíquico e nos processos psicológicos e psicopatológicos do indivíduo, norteado por uma concepção de sujeito abstrato e descontextualizado historicamente. Por sua vez, as práticas clínicas emergentes (Dutra, 2004), apontam para um maior interesse e articulação entre a clínica e o social. Isto implica significativas alterações na concepção de sujeito e, consequentemente, novas interpretações das teorias psicoterápicas.
}

na Política Nacional de Humanização (PNH). Como objetivo, apresentaremos uma proposta para o desenvolvimento de um estágio supervisionado em Psicologia que prepare os psicólogos para a atuação não só na clínica privada, mas nos serviços públicos de saúde em seus diversos níveis de atenção.

Esta proposta pode ser adaptada a qualquer abordagem psicológica, entretanto, nesta experiência, vimos que a ACP, por seus princípios humanistas-existenciais, coadunados com os princípios da $\mathrm{PNH}$, facilitará a atuação do psicólogo na saúde pública/coletiva de forma mais apropriada.

\section{Fundamento}

O primeiro argumento: a PS é definida por Alves (2008), como um campo de aplicação da Ciência Psicologia dedicado aos cuidados de saúde geral. Sua prioridade assenta-se sobre as assistências de promoção de saúde e de prevenção de doenças. Nesse enfoque, a intervenção do psicólogo se alarga para além das práticas clínicas e da saúde mental, já que o objetivo maior é a saúde global das pessoas inseridas em seus coletivos sociais.

Esta definição permite mostrar uma aplicação da Psicologia que se amplia para admitir outras possibilidades de intervenção em saúde para além da clínica. Entende-se melhor tal argumento quando pensamos em níveis de atenção em saúde. Tomaremos por base as investigações de Alves, Eulálio \& Brobeil (2009) para delimitar as ações do psicólogo nos diversos níveis de atenção. O nível de Atenção Básica (primário) é o lugar de fazer a prevenção primária que deve estar diretamente relacionada e condicionada à promoção da saúde. Suas ações não devem dirigir-se a um indivíduo, senão aos planos de educação para a saúde, os quais devem ter, como suporte, conteúdos das demais áreas de aplicação da Psicologia na interface com outras profissões de saúde. Tem como característica central a atuação nos problemas epidemiológicos da população beneficiária e investe na construção de estilos de vida saudáveis e na prevenção de comportamentos de risco. $\mathrm{O}$ objetivo é fazer com que os novos comportamentos saudáveis sejam duradouros e utilizados durante toda a vida. Assim, a prevenção primária deverá ser realizada antes que se encontre um problema concreto. Para tal intervenção o conhecimento epidemiológico prévio do coletivo social a ser assistido deverá ser utilizado como guia das ações de saúde. Numa palavra: seria trabalhar com as possibilidades de que possa aparecer uma enfermidade.

O nível de Atenção Especializada (secundário) é o tradicionalmente mais conhecido e desenvolvido tanto da Psicologia como das especialidades médicas em geral. Em termos da Psicologia, possui um fundamento teórico/prático bem sedimentado na clínica, dedicado à saúde mental e à psicoterapia. Contraditoriamente, é o campo 
mais problemático quando se propõe sua aplicação em saúde pública/coletiva. Inúmeras investigações concluem que existe uma sobreutilização das intervenções especializadas no nível de Atenção Primária (AP), sem que os psicólogos percebam a inadequação entre suas práticas e seu nível de aplicação. Campos \& Guarido (2007) e Dimenstein (2003), por exemplo, asseguram que a dedicação a $80 \%$ das atividades dos psicólogos de AP à psicoterapia individual lhes impede de desenvolver outras atividades importantes e mais apropriadas ao primeiro nível de atenção de saúde.

O nível de Atenção de Alta Complexidade (terciário) está dedicado à assistência aos problemas de alta complexidade derivados dos outros níveis de atenção $\left(1^{\circ} \mathrm{e}\right.$ $2^{\circ}$ ) e com as pesquisas em saúde (Andrasik, Otis, Turner, \& Simón, 1999). Em geral são realizadas nos hospitais (Yamamoto \& Cunha, 1998). Entretanto, pode também ser feita nos ambulatórios. A prevenção terciária inclui o seguimento de pacientes em tratamento clínico, cirúrgico (Antón \& Méndez, 1999), quimioterápico e radioterápico. Os cuidados paliativos são também aplicáveis a este nível de atenção (Alves, 2008).

Dito isto, passamos ao segundo argumento: a ACP. Esta se inicia com Carl Rogers em meio à década de 30 do século XX. O seu pensamento que, naturalmente, incidiu sobre sua produção, inclui uma grande quantidade de ideias e questões sobre a natureza do Homem, a educação, a abordagem de grupo, dentre tantas outras, e vão muito além dos limites da Teoria da Psicoterapia Centrada no Cliente. A este leque mais amplo de ideias, capazes de organizar a experiência de forma bem sucedida, Rogers chamou de Abordagem Centrada na Pessoa (Wood, 1994).

A ACP não é uma teoria, mas um modo de ser que tem em sua base princípios norteadores como respeito pelo indivíduo, por sua autonomia e dignidade; crença na tendência formativa direcional das pessoas; perspectiva de vida positiva; intenção de ajudar o ser humano a fazer mudanças construtivas na personalidade; flexibilidade do pensamento e ação; tolerância quanto às incertezas e ambiguidades (Wood, 1994). A ACP foi uma expressão utilizada por Rogers (1977/1986) e representa uma evolução em seu pensamento que sai do campo exclusivo da psicoterapia para uma forma ou uma filosofia de trabalho que pode se aplicar aos vários setores da saúde, da educação, do trabalho, do aconselhamento familiar e entre tantos outros campos. Tal posicionamento, norteador do modo de pensar e se posicionar no mundo, pode abrigar os novos paradigmas da saúde e da clínica, sobretudo pela afinidade de princípios de valorização do humano e da vida.

Acionamos a ACP para ancorar a proposta sobre a formação do psicólogo para atuar na saúde pública, fazendo ao mesmo tempo ponte com a $\mathrm{PNH}$, porque o contexto dessa Abordagem nos conduz à pretensão de unir seus ideais à política de humanização do SUS, já que os princípios da ACP seriam o fio condutor capaz de perpassar todas as ações de atenção à saúde, de preparação e capacitação dos profissionais, de sensibilização dos atores (agentes e pacientes) envolvidos, tanto no processo de compreensão do que seja humanizar serviços, como para auxiliar na criação de novos valores capazes de gerar a mudança de comportamento entre os profissionais de saúde, pretendida pelo SUS.

\section{A ACP na interface com a Política Nacional de Humanização do SUS}

A PNH surgiu com o intuito de fortalecer o SUS, em seus níveis de atenção e de gestão, no que se refere ao lidar com as questões sociais e subjetivas. Pois, apesar das conquistas alcançadas pelos princípios da universalização, equidade, integralidade, participação popular e descentralização (Ministério da Saúde, 2000), existe ainda certa fragilidade nas relações entre os protagonistas responsáveis pela funcionalidade efetiva dos Serviços. Assim, a PNH nasceu da necessidade de fortalecer o trabalho em equipe e as relações entre os diferentes profissionais de saúde, bem como entre estes e os usuários (Ministério da Saúde, 2004b).

Essa política pressupõe um investimento no desenvolvimento de atitudes de corresponsabilidade, autonomia, protagonismo, ampliação das trocas de saberes, estreitamento dos vínculos interprofissionais e interpessoais, estabelecimento de vínculos solidários e a participação coletiva no processo de gestão. Tudo isso com o objetivo de construir uma assistência em saúde pautada na integralidade, no respeito, no diálogo, na qualidade do atendimento e na valorização do humano e da vida (Ministério da Saúde, 2004b). O documento que trata da PNH, da atenção e gestão do SUS (Ministério da Saúde, 2004a) argumenta que, quando se avalia um Serviço, um dos aspectos que mais tem chamado a atenção é o despreparo dos profissionais para lidar com a dimensão subjetiva que toda prática de saúde supõe. Tal dimensão subjetiva é difícil de atender porque remete ao desprendimento de antigas atitudes para dar lugar à formação de novos valores. Isto exige, pois, uma formação não apenas teórica, mas voltada à sensibilização para a aquisição e consolidação de novos valores.

O cenário indica, então, a necessidade de mudanças. Mudanças no modelo de atenção que não se farão sem que ocorram também mudanças no modelo de gestão (Ministério da Saúde, 2004a). De acordo com o referido documento, por humanização entende-se a valorização dos diferentes sujeitos implicados no processo de produção de saúde: usuários, trabalhadores e gestores. Os valores que norteiam esta política são a autonomia e o protagonismo dos sujeitos, a co-responsabilidade entre eles, o estabelecimento de vínculos solidários e a participação coletiva no processo de gestão. 
Benevides \& Passos (2005), em avaliação de programas de humanização em vários Serviços, viram que havia projetos, atividades, propostas, mas em todos era evidente o caráter fragmentado dessas iniciativas, não só na relação de baixa horizontalidade que se verificava entre elas, mas, também no modo vertical como elas se organizavam dentro do Ministério da Saúde e do SUS. Para eles há um duplo problema: seja o da banalização do tema da humanização, seja o da fragmentação das práticas ligadas a diferentes programas de humanização da saúde. Trata-se de um mesmo problema em uma dupla inscrição teórico-prática, que leva à necessidade de construir uma política pública eficaz e transversal de humanização da/na saúde.

Diante desses pressupostos da política de humanização do SUS, vemos que é perfeitamente possível relacioná-los com os princípios norteadores da ACP. Pois, como enfatiza Menezes (2007), a atitude humanizadora ocorre quando se apreciam as relações interpessoais e se consegue enxergar e valorizar o ser humano como um ser de possibilidades, um ser com potencial para o crescimento e para a autonomia. Um sistema de saúde que preza pela autenticidade de seus profissionais e usuários, valorizando-os de modo integral, buscando compreendê-los empaticamente e os aceitando incondicionalmente colabora positivamente no processo de produção de saúde, mobiliza os sujeitos sociais a transformarem realidades, o que, consequentemente, acarreta transformações neles próprios (Ministério da Saúde, 2004a).

Segundo Bacellar, Rocha \& Flôr (2012), as atitudes de compreensão empática, aceitação positiva incondicional e congruência, podem contribuir de forma significativa para a minimização dos obstáculos de implantação de um conceito e de uma prática de saúde mais ampla, permitindo uma maior integração e crescimento coletivo. Mediante a aplicação dos princípios que regem a ACP o psicólogo pode atuar como mediador das ações das equipes de saúde, levando-as a entenderem a filosofia do cuidado humanizado prescrita pelo SUS. A sintonia entre os preceitos e práticas específicas da ACP com as prerrogativas da PNH pode auxiliar a pôr em prática os princípios do SUS sobre a necessária humanização dos serviços de saúde.

\section{Proposta para um estágio supervisionado em psi- cologia}

Na tentativa de contribuir com este debate, que tem na crítica à formação dos psicólogos o seu eixo central, apresentamos estas ideias que formam parte de uma proposta de estágio (obrigatório) supervisionado em psicologia, que vimos pondo em prática desde o ano de 2009, no curso de Psicologia da Universidade Estadual da Paraíba (UEPB). Oficialmente, o estágio curricular da UEPB ainda é centrado no modelo da clínica privada e realizado basicamente no âmbito físico da Clínica Escola. Na proposta aqui apresentada, o estágio se organiza a partir dos níveis de intervenção e, assim, deve ser realizado tanto na Clínica Escola da UEPB como nos vários setores em que se pode construir a saúde pública/coletiva.

\section{Descrição do grupo}

Os grupos são formados por um professor supervisor e quatro a oito estudantes. Tal supervisão é oferecida no $5^{\circ}$ ano da formação acadêmica em Clínica de base Humanista-existencial Centrada na Pessoa.

\section{0 estágio supervisionado}

A supervisão é essencial para toda atividade de estágio, uma vez que permite ao estudante, suporte e orientação no início do desenvolvimento de suas práticas profissionais. Os encontros de supervisão duram de 12 a 16 horas por semana, a depender do número de supervisionandos e são distribuídos em dias alternados. São realizados basicamente em grupo e, ocasionalmente, individualmente, a depender das necessidades do supervisor ou dos supervisionandos. A metodologia de trabalho ampara-se nas três funções realçadas por Buys (1987): suporte teórico, técnico e experiencial.

A supervisão inclui discussões, avaliações e reflexões sobre as experiências dos supervisionandos. São realizados também seminários temáticos, discussão de filmes, e algumas vezes discussões informais sobre política partidária local, nacional e internacional, história e arte, dentre outros temas. É importante ressaltar que a técnica de role-playing ${ }^{2}$ é também utilizada para o exercício das escutas psicológicas e análises dos sonhos.

O conteúdo teórico da supervisão deve ser mínimo e deve atender principalmente aqueles temas que deixaram de ser privilegiados no processo de formação. Nesta experiência, temos de centrar o investimento nos conteúdos da Psicologia da Saúde, de Saúde pública/coletiva, e, sobretudo, no estudo das políticas e programas do SUS e SUAS. Tal medida justifica-se pelo fato de que o PPP do curso em tela ainda apresenta lacunas quanto aos aspectos teóricos elencados aqui. Em relação à supervisão dos casos clínicos atendidos em psicoterapia na Clínica Escola, a supervisão aporta o suporte ao supervisionando, o que lhe permite melhorar seu desempenho psicoterapêutico. Buys (1987) descreve a supervisão como o momento “...em que se fala sobre a psicoterapia que está sendo feita pelo supervisando [sic], onde a experiência

\footnotetext{
O role-playing ou Jogo de papéis é uma técnica do psicodrama que permite explorar e treinar de forma didática as possibilidades de representação de um determinado papel (Francischetti, Corrêa, Vieira, Lazarini, Rolin, \& Soares, 2011). A utilização dessa técnica na formação do psicoterapeuta permite ao aluno experienciar e avaliar sua conduta no desempenho do papel profissional.
} 
psicoterapêutica é examinada, questionada, melhor entendida, enfim, onde o psicoterapeuta iniciante reflete junto com o supervisor sobre sua experiência como psicoterapeuta" (p.23).

\section{Instituições e programas onde são desenvolvidos os estágios}

Os locais e Programas em que são desenvolvidos o estágio com suas respectivas atividades incluem a Clínica Escola de Psicologia da UEPB, Centros de Saúde, Hospitais, a Maternidade Elpídio de Almeida, Centros de Referência de Assistência Social (CRAS), Centros de Referência Especializada de Assistência Social (CREAS), Unidades Básicas Saúde da Família (UBSF), Escolas municipais e estaduais, Centro de Educação de Jovens e Adultos, Centro de Atenção Psicossocial (CAPS), Núcleos de Assistência à Saúde da Família (NASF), dentre outros.

É fundamental que a ideia de atuação do psicólogo seja compreendida a partir das prerrogativas da Psicologia da Saúde. Ou seja, como uma ação voltada à saúde geral, tal como foi explicado na primeira parte deste trabalho. Nesta direção, é preciso localizar e associar, de forma dinâmica e não dicotômica, os níveis de atenção à saúde e suas respectivas intervenções. Na atenção básica/primária, através das ações de prevenção de doenças e promoção da saúde. Ação que deve ser realizada em consonância com as equipes multiprofissionais de saúde dos NASFs, ouvidas as equipes dos CRAS. Na proposta de humanização dos serviços públicos (hospitais, centros de saúde, equipes de saúde da família, NASFs, UPAs, SAMU). Na atenção especializada/secundária (Ambulatórios e clínicas privadas). Na atenção de alta complexidade (Nos hospitais).

A diversidade de locais para o desenvolvimento do estágio é possível porque a UEPB tem um programa de formação de estudantes, o Estágio Multidisciplinar Interiorizado - EMI, que permite o deslocamento dos supervisionandos para outras localidades do Estado. O EMI é uma atividade curricular obrigatória, realizada em municípios paraibanos conveniados à UEPB. Tal convênio abrange Secretarias de Saúde (Programas do SUS: UBSF, NASF, CAPS e hospitais), Secretarias de Educação (Escolas Municipais de Ensino Fundamental e Médio), Secretarias de Assistência Social (Programas do SUAS: CRAS e CREAS), bem como domicílios urbanos e rurais. O EMI cumpre um papel fundamental para a consecução desta proposta, pois a maioria das atividades, nele realizadas, só é possível porque os estudantes tem acesso aos setores de saúde, de assistência social e de educação. A Clínica Escola da UEPB, em sua limitação natural, apenas permite a apreensão de uma parte da experiência exigida para a formação, a saber: a formação para a psicoterapia.

\section{Exigências para o preparo teórico}

Os temas devem ser eleitos no início de cada unidade temática de acordo com avaliações realizadas pelo grupo, das necessidades e das demandas. Os princípios fundadores da ACP e a teoria da psicoterapia são apenas revisitados, já que, no curso em destaque, o $4^{\circ}$ ano de formação acadêmica inclui uma disciplina específica dedicada ao estudo dessa abordagem. As principais temáticas são: Psicologia da saúde; estudo das Políticas e programas do SUS e do SUAS; saúde pública/coletiva; a clínica ampliada; psicopatologias; psicofarmacologia; cuidados paliativos; psicologia nas emergências e desastres; teoria e análise dos sonhos; arteterapia; ludoterapia; escutas psicológicas e as temáticas referentes aos casos clínicos em acompanhamento. Podemos destacar como os mais recorrentes: depressão; suicídio; transtorno do pânico; distúrbio de comportamento; comportamentos aditivos ao álcool e outras drogas; ansiedade; dislexia e o déficit de atenção.

\section{Proposta de atividades}

\subsection{Plantões de triagem}

A clínica-escola de Psicologia da UEPB é conveniada ao SUS e oferece o serviço de triagem, em regime de plantões rotativos. Esses propõem uma forma de cadastro e levantamento dos perfis dos usuários da clínica-escola. Para o alcance de tal propósito, a triagem faz uso de uma entrevista semiestruturada que permite um maior conhecimento da pessoa e dos fatores que motivaram a procura. Essa entrevista inicial comporta a coleta de informações importantes, tais como a queixa principal, os dados sociodemográficos, a estrutura familiar, as experiências anteriores com atendimentos psicológicos e as expectativas para com os serviços ofertados pela clínica escola.

A triagem além de servir à elaboração do cadastro e coleta de dados serve, também para a realização do acolhimento atendendo as prerrogativas da PNH. Através dela é feita uma escuta, não apenas dos sintomas apresentados pela pessoa, mas também de seus sentimentos e sofrimentos. Perfeito \& Melo (2004) acrescentam que a triagem permite ir além dos sintomas, das queixas, das designações nosológicas, porque proporciona a compreensão da pessoa inserida no contexto de seu grupo de pertença. E, como enfatiza Rocha (2011), a triagem permite avaliar e compreender a necessidade do atendimento, as expectativas do cliente e as possibilidades de encaminhamento. É o momento de esclarecer os serviços oferecidos pela clínica-escola e também funciona como um dos passos iniciais para o início de uma psicoterapia. 


\subsection{Plantão de escuta psicológica}

O Conselho Federal de Psicologia (conforme citado por Mahfoud, 1999) afirma que o Plantão de Escuta Psicológica é uma proposta inovadora classificada como uma técnica alternativa emergente. No plantão psicológico, o profissional permanece à disposição da população em horário predeterminado e ininterrupto. O objetivo principal é auxiliar a pessoa que busca esse serviço no momento de sua necessidade (Tassinari, Formiga, Mota, Souza \& Silva, 2005). A escuta psicológica visa contribuir para uma compreensão mais ampla da pessoa e da sua situação, não focando apenas a catarse, apesar dessa acontecer durante o encontro. Esse encontro pode ocorrer em uma ou mais consultas sem duração preestabelecida, podendo a pessoa ser encaminhada para outros serviços, inclusive para a psicoterapia. Contudo, tal encaminhamento deve ser objetivo secundário da escuta.

O Plantão de escuta psicológica é uma modalidade de ação que proporciona aos estudantes uma experiência enriquecedora para o seu amadurecimento profissional, uma vez que, como afirma Cury (1999), possibilita o acesso a uma diversidade de problemas, levando a um contato direto com o inesperado. Esta intervenção é adequada à atenção primária, já que contribui para a promoção de saúde (Tassinari et al., 2005). $\mathrm{O}$ atendimento imediato à pessoa, no momento de uma crise, estimula-a às práticas de cuidado consigo mesma, atingindo, assim, os objetivos da prevenção primária.

\subsection{Psicoterapia Individual}

A psicoterapia, regida pelos princípios da ACP, se fundamenta na crença de que o ser humano tem uma tendência intrínseca para o crescimento e para a atualização. Parte da valorização e da confiança nas pessoas e em suas capacidades de explorar e compreenderem a si mesmas, de solucionar seus problemas, escolherem seus próprios caminhos e avaliar seus progressos em relação a suas escolhas (Rogers \& Kinget, 1951/1977). Na psicoterapia o papel do terapeuta é o de facilitador do processo de autoconhecimento e crescimento pessoal da pessoa. Essa facilitação dá-se pelo estabelecimento de uma atmosfera de calor e segurança, regida pelas atitudes de aceitação positiva incondicional, compreensão empática, congruência e autenticidade. Para Rogers, o sucesso da terapia não está relacionado ao uso de técnicas, e sim, à qualidade da relação estabelecida entre o terapeuta e o cliente (Rogers \& Kinget, 1951/1977).

A clínica-escola de Psicologia oferece o atendimento de psicoterapia individual na perspectiva Centrada na Pessoa, para crianças, adolescentes e adultos. No período de estágio correspondente à experiência aqui relatada, as principais demandas e queixas apresentadas foram: depressão; síndrome do pânico; conflitos interpessoais e familiares; ideações suicidas; problemas de socialização; baixa autoestima; estresse; estafa física e mental; problemas de ordem sexual; indecisão e dificuldades na escolha profissional; transtornos de ansiedade; instabilidade emocional; luto não elaborado; sentimentos de incapacidade e solidão; dislexia; déficit de atenção; distúrbio do comportamento; falta de sentido na vida; bullying na escola; tabagismo; busca por autoconhecimento e amadurecimento pessoal.

\subsection{Palestras}

A saúde também pode ser trabalhada por meio de palestras que proporcionam valiosas reflexões e discussões com a comunidade. Elas podem ser desenvolvidas em diversos locais e serviços de educação e saúde e podem abordar múltiplas temáticas, que variam a partir da observância das necessidades emergentes. Nas nossas experiências alguns temas são recorrentes, como: os valores morais e as relações interpessoais em sala de aula; a importância da família na relação família-escola; bullying; síndrome de burnout: sinais de alerta e modos de prevenção; higiene pessoal e mental; benefícios do aleitamento materno; depressão pós-parto; qualidade de vida; DSTs; reprodução e métodos contraceptivos; planejamento familiar; vasectomia e saúde do homem; o envelhecimento saudável, dentre outros.

\subsection{Atendimento domiciliar}

Os atendimentos domiciliares são realizados pelos supervisionandos aos usuários dos serviços de saúde que, por alguma razão, são impossibilitados de se deslocarem de suas casas para irem aos locais de atendimento. A maioria das pessoas que têm acesso a essa modalidade de assistência psicológica são idosas, com dificuldades de locomoção e com outras particularidades como a depressão, acidente vascular encefálico, doenças relacionadas com a idade, solidão, baixa autoestima e pensamentos negativos diante da vida.

\subsection{Atividades educativas}

As atividades educativas são realizadas em caráter interdisciplinar e multidisciplinar. São desenvolvidas em diversos locais como escolas, CAPSs, maternidade, dentre outros. São viabilizadas mediante oficinas, panfletagens e dinâmicas que trabalharam questões como: importância do toque e do carinho para o vínculo mamãe-bebê; orientação a jovens sobre como fazer o autoexame da mama; a relação entre a alimentação materna e o aleitamento; as posições corretas para o aleitamento materno e os benefícios desse alimento para o bebê; ati- 
vidades musicais e de alfabetização com os usuários do CAPS; atividades musicais com crianças atendidas pelo Programa de Erradicação do Trabalho Infantil (PETI); oficinas sobre a violência na escola e o fenômeno do Bullying; e atividades de sondagem de conhecimentos com crianças do ensino fundamental sobre a higiene pessoal e mental. Todas essas atividades proporcionam ricas reflexões, construções e trocas de conhecimentos entre os estagiários e a comunidade.

\subsection{Formação de grupos terapêuticos}

Os grupos terapêuticos podem ser formados em diferentes Serviços e sua funcionalidade assemelha-se ao Plantão de Escuta Psicológica. Em nossas experiências temos formado grupos de homens usuários do CAPS III (Unidade de referência para usuários portadores de transtornos mentais no momento de crise psiquiátrica), que apresentavam diagnósticos bem diversificados como esquizofrenia, transtorno bipolar, alcoolismo, depressão e fobia social. Os grupos de gestantes proporcionavam o compartilhamento das experiências, das angústias e das ansiedades relativas ao período de gestação, parto e puerpério. Os grupos de idosos possibilitavam um espaço, no qual esses podem falar sobre suas preocupações e sentimentos adjacentes ao processo de envelhecimento, principalmente, os sentimentos relacionados às mudanças de hábitos consequentes do diagnóstico de doenças crônicas, como hipertensão e diabetes.

\subsection{Orientação às famílias}

Em nossa experiência são recorrentes as orientações às famílias dos pré-adolescentes em atendimento de psicoterapia na clínica-escola da UEPB. Orientações de caráter multidisciplinar também são realizadas com grupos de mães primíparas, numa maternidade pública da cidade, e as orientações versam sobre aleitamento materno e os significados dos choros dos bebês. Também são comuns as orientações a grupos de mães de crianças, atendidas nas Unidades Básicas de Saúde da Família (UBSFs) que apresentavam dificuldades na escola e/ou problemas no desenvolvimento motor e cognitivo.

\subsection{Interconsulta entre equipe multiprofissional de saúde}

A interconsulta entre equipe multiprofissional de saúde é definida por Botega (2006) como um tipo de metodologia usada pelo psicólogo para desenvolver a compreensão e melhorar a assistência ao usuário. Para atingir esse fim, utiliza-se o diagnóstico e o tratamento das questões psicológicas, problemas interpessoais, dificul- dades institucionais relacionados ao usuário, à família e à equipe de saúde. Trata-se de uma atividade relevante para os profissionais da saúde, tendo em vista que proporcionará um novo olhar acerca dos usuários e de seus contextos psicossociais.

\subsection{Diálogo multiprofissional com equipes de trabalho do CRAS e CREAS}

Assim como a interconsulta multiprofissional com equipes de saúde, o diálogo entre os psicólogos e as equipes dos programas de assistência social, tais como CRAS e CREAS, é extremamente importante, uma vez que a Psicologia e a Assistência Social são áreas afins, que lidam com as mesmas problemáticas como, por exemplo, os mais variados tipos de violência (doméstica, sexual, psicológica etc.), muitas vezes encontrados nas famílias. Trata-se de uma aproximação necessária da Psicologia com essas problemáticas e com as pessoas nelas inseridas.

\subsection{Orientações e encaminhamentos de pessoas a outros programas de assistência social}

Aqui o trabalho dirige-se à identificação das necessidades individuais e/ou coletivas dos assistidos no sentido de provê-los de informações sobre os programas de assistência social e realizar os encaminhamentos pertinentes a cada caso. Macedo \& Dimenstein (2012) afirmaram que os psicólogos têm hoje no Brasil um campo de inserção consolidado junto aos serviços de saúde pública e Assistência Social. Portanto, é necessário que o psicólogo conheça os propósitos e as dinâmicas de atuação desses programas e tenha uma boa articulação com cada um deles. Nesse contexto, o momento de identificação das demandas e das necessidades é fundamental para esta ação, já que estas pertencem a cenários bem diversificados, tais como uma orientação para buscar um curso de formação com vistas ao provimento da necessidade de trabalho e renda familiar, a orientação dirigida aos problemas escolares/educacionais desses coletivos, orientações para a saúde geral e mental, bem como orientações e encaminhamentos para resolver problemas de ordem jurídica como: violência doméstica e de gênero, maus-tratos, criminalidade e outros.

\subsection{Atividades multidisciplinares}

O Estágio Multidisciplinar Interiorizado (EMI) permite a interação entre os estudantes do último ano de formação dos diferentes cursos da área de saúde, tais como Psicologia, Odontologia, Fisioterapia, Farmácia e Enfermagem. Possibilita a troca de conhecimentos 
e as práticas multidisciplinares proporcionando um maior aprendizado tanto em relação ao trabalho em equipe, quanto às atividades que são desempenhadas pelo grupo. As atividades multidisciplinares, geralmente, apresentam uma proposta educativa valorizando a integração dos pontos de vista dos diferentes campos do saber em relação às temáticas abordadas. Durante o EMI formam-se grupos de caráter multidisciplinar para trabalhar com gestantes, idosos, crianças e assim possibilitar a prestação de uma assistência em saúde mais completa.

Na experiência em tela, organizamos atividades multidisciplinares com todos os estagiários nos grupos de idosos e gestantes do CRAS, onde foram realizadas palestras rotativas a cada semana na área de cada estagiário com assuntos pertinentes a cada grupo, como saúde bucal do bebê (Odontologia), cuidados pós-parto (Enfermagem), medicações pertinentes à gestante (Farmácia), depressão pós-parto (Psicologia), exercícios para facilitar o período de gestação e a hora do parto (Fisioterapia), atividades de respiração e alongamento com grupo de idosos (Fisioterapia), serviços de saúde oferecidos pelos PSF's aos idosos (Enfermagem), a velhice e seus agravantes para a saúde (Psicologia).

\subsection{Atuar como mediador pedagógico junto à equipe multiprofissional}

Mediante a aplicação dos princípios que regem a ACP o psicólogo pode atuar como mediador das ações das equipes de saúde através de rodas de conversa, oficinas temáticas, discussão de textos, sensibilização, dentre outras. A função de mediação pedagógica possibilita a reflexão e a compreensão da filosofia do cuidado humanizado, prescrita pelo SUS. A sintonia entre os preceitos e práticas específicas da ACP com as prerrogativas da PNH, pode ajudar a pôr em prática os princípios do SUS sobre a necessária humanização dos serviços de saúde. Pode também auxiliar no processo de construção de novos valores relacionados à autonomia e ao protagonismo dos sujeitos, a corresponsabilidade entre eles, o estabelecimento de vínculos solidários e a participação coletiva no processo de gestão.

\section{Considerações finais}

A proposta apresentada parte da crítica à formação do psicólogo, ainda tradicional, e voltada, basicamente, para atividade clínica-psicoterapêutica. Fizemos uma reflexão sobre a sua formação e propusemos algumas ações que prometem aportar um incremento às práticas de saúde voltando-as para a necessidade de realizar um trabalho mais apropriado à intervenção em saúde pública/coletiva.
A ideia é preparar melhor os futuros profissionais antes que se dê sua inserção na Rede pública de atenção à saúde, para uma prática que se amplie para além da atividade psicoterapêutica, já que esta tem sido praticada de modo equivocado, quando não se observa a extensão das ações do psicólogo nos diversos programas governamentais. Aqui, o trabalho do psicólogo é apresentado como capaz de ser desenvolvido em instituições públicas de saúde e educação, bem como em domicílios urbanos e rurais, com o objetivo de instigar nos estagiários o desenvolvimento das diversas habilidades para trabalhar nos serviços de saúde, sejam eles públicos ou privados, possibilitando que esses, ainda na graduação, possam ter um maior contato com a realidade de trabalho de sua profissão. Assim, a atividade do profissional de Psicologia ultrapassa em muito as ações clínicas tradicionais. Trata-se de um trabalho realizado em vários níveis de ação. No plano das ações clínicas, se aplica a uma visão de clínica ampliada, sem, contudo, abandonar a clínica especializada. Esta, que jamais pode ser negligenciada, deve ser utilizada quando a sua indicação for comprovada. Não pode ser utilizada de forma indiscriminada e generalizada e, ainda, tal como muitas vezes ocorre, para dissimular a falta de clareza da ação em outros níveis de atenção à saúde.

Há uma necessidade real de que as unidades formadoras busquem garantir uma formação menos elitizada e mais engajada com as políticas públicas. Uma formação mais moderna e que valorize o dinamismo na mudança dos perfis de saúde das populações e sua relação com a emergência de novas Políticas e Programas do SUS e do mercado de trabalho do psicólogo.

Os apontamentos aqui propostos visam levar os psicólogos a realizarem uma mudança de foco da ação, mostrando que é preciso avançar na concepção de formação na medida em que saímos do modelo mais tradicional, onde a linguagem é mais limitada, para um modelo de atuação mais amplo que permite juntar abordagens tradicionais com as novas configurações e demandas de atuação exigidas no momento atual. E privilegiando as ações que tradicionalmente definiram a Psicologia, sem perder de vista o cenário atual das demandas de ações advindas do mercado de trabalho que ora se abre para o psicólogo nas Políticas e Programas governamentais.

\section{Referências}

Alves, R. F., Eulálio, M. C. \& Brobeil, S. A. J. (2009). La promoción de la salud y la prevención de enfermedades como actividades propias de labor de los psicólogos, Arquivos Brasileiros de Psicologia. 61(2), 1-12.

Alves, R. F. (2008). Intervenciones de profesionales en el campo de la salud: estudio antropológico comparativo en Brasil, España y Portugal. Tese de Doutorado, Universidade de Granada, Granada. 
Andrasik, F., Otis, J., Turner, B. \& Simón, M. A. (1999). Estrategias de investigación en psicología de la salud. In M. A. Simon (Org.), Manual de psicología de la salud: fundamentos, metodología y aplicaciones (pp. 259-306). Madrid: Editorial biblioteca Nueva.

Antón, D. M. \& Méndez, F. X. (1999). Líneas actuales de investigación em psicología de la salud. In M. A. Simon (Org.), Manual de psicología de la salud: fundamentos, metodología y aplicaciones (pp. 217-256). Madrid: Editorial biblioteca Nueva.

Bacellar, A., Rocha, J. S. X. \& Flôr, M. S. (2012). Abordagem Centrada na Pessoa e Políticas Públicas de Saúde Brasileiras do século XXI: Uma aproximação possível, Revista do NUFEN, 4(1), 127-140.

Benevides, R. \& Passos, E. (2005). A humanização como dimensão pública das políticas de saúde. Ciência e Saúde coletiva, 10(3), 561-571.

Bock, A. M. B., Furtado, O. \& Teixeira, M. L. T. (2002). A evolução da ciência psicológica. In A. M. B. Bock, O. Furtado \& M. L. T. Teixeira. Psicologias: uma introdução ao estudo de psicologia (pp. 31-44). São Paulo: Saraiva.

Botega, N. J. (2006). Prática Psiquiátrica no Hospital Geral: Interconsulta e Emergência. Porto Alegre: Artmed.

Buys, R. C. (1987). Supervisão de psicoterapia na Abordagem centrada na Pessoa. São Paulo: Summus.

Campos, F. C. B. \& Guarido, E. L. (2007). O psicólogo no SUS: suas práticas e as necessidades de quem o procura. In M. J. P. Spink (Org.), A psicologia em diálogo com o SUS: prática profissional e produção acadêmica (pp. 81-103). São Paulo: Casa do Psicólogo.

Cury, V. E. (1999). Plantão Psicológico em clínica-escola. In M. Mahfoud (org.), Plantão Psicológico: Novos horizontes (pp.115-134). São Paulo: Editora C.I.

Dimenstein, M. (2003). Los (des)caminos de la formación profesional del psicólogo en Brasil para la actuación en la salud pública. Revista Panamericana de Salud Pública, 13(5), 341-345.

Dutra, E. (2004). Considerações sobre as significações da psicologia clínica na contemporaneidade. Estudos de Psicologia (Natal), 9(2), 381-387.

Figueiredo, L. C. M. (2010). Matrizes do pensamento psicológico. Petrópolis: Vozes.

Francischetti, I., Corrêa, A. C. L., Vieira, C. M., Lazarini, C. A., Rolin, L. M. G., \& Soares, M. O. M. (2011). Role-playing. Estratégia inovadora na capacitação docente para o processo tutorial, Interface - Comunicação, Saúde, Educação. 15(39), 1207-1218.

Lo Bianco, A. C., Bastos, A. V. B., Nunes, M. L. T., \& Silva, R. C. (1994). Concepções e atividades emergentes na psicologia clínica: implicações para a formação. In Conselho Federal de Psicologia (Org.), Psicólogo brasileiro: práticas emergentes e desafios para a formação (pp. 7-76). São Paulo: Casa do Psicólogo.
Macedo, J. P., \& Dimenstein, M. (2012). O trabalho dos psicólogos nas políticas sociais no Brasil. Avances en Psicología Latinoamericana, 30(1), 182-192.

Macedo, J. P., \& Dimenstein, M. (2011). Expansão e Interiorização da Psicologia: Reorganização dos Saberes e Poderes na Atualidade. Psicologia: Ciência e Profissão, 31(2), 296-313.

Mahfoud, M. (1999). Introdução: Frutos Maduros do Plantão Psicológico. In M. Mahfoud (Org.), Plantão Psicológico: Novos horizontes. (pp. 11-14). São Paulo: Editora C.I.

Menezes, A. L. S. (2007). Humanização em Saúde: A abordagem Centrada na Pessoa. Boletim do Instituto de Psicologia/UFRJ, 21(38). Recuperado em 11 de agosto de 2013, de http://www.psicologia.ufrj.br/boletimip/index2. php?option=com_content\&do_pdf=1\&id=35.

Ministério da Saúde (2000). Sistema Único de Saúde (SUS): princípios e conquistas. Brasília: Ministério da Saúde.

Ministério da Saúde (2004a). Humaniza SUS: Política Nacional de Humanização da atenção e gestão do SUS. Brasília: Ministério da Saúde.

Ministério da Saúde (2004b). Humaniza SUS: Documento base para Gestores e Trabalhadores do SUS. Brasília: Ministério da Saúde.

Ministério do Desenvolvimento Social e Combate à Fome. (2011). Censo SUAS 2009 - CREAS. Brasília: Secretaria de Avaliação e Gestão da Informação.

Perfeito, H. C. C. S. \& Melo, S. A. (2004). Evolução dos professos de triagem psicológica em uma clínica escola. Rev. Estudos de Psicologia, 21(1), 33-42.

Rocha, M. C. (2011). Plantão psicológico e triagem: aproximações e distanciamentos. Revista do NUFEN, 3(1).

Rogers, C. R. \& Kinget, G. M. (1951/1977). Psicoterapia e Relações Humanas. Teoria e prática da terapia não-diretiva. Belo Horizonte: Interlivros.

Rogers, C. R. (1977/1986). Sobre o poder pessoal. São Paulo. Martins Fontes.

Tassinari, M. A., Formiga, A., Mota, R. P., Souza, L. S. L. \& Silva, T. L. (2005). O Plantão Psicológico como porta de entrada do Serviço de Psicologia Aplicada: desenvolvimento dos estagiários, a partir da versão de sentido. Trabalho apresentado no VI Fórum Brasileiro da Abordagem Centrada na Pessoa, Canela, Brasil.

Wood, J. K. (1994). Prólogo. In J.K.Wood, M.L. Assumpção, M. A. Tassinari, M. Japur, M. Serra, M. \& R. Rosenthal (Orgs), Abordagem Centrada na Pessoa. Vitória: Editora Fundação Ceciliano Abel de Almeida/UFES.

Yamamoto, O. H. \& Cunha, I. M. F. F. O. (1998). O psicólogo em hospitais de Natal: uma caracterização preliminar. Psicologia, Reflexão e Critica, 11(2), 345-362. 
Railda Fernandes Alves - Doutora em Antropologia da Saúde pela Universidade Estadual da Paraíba (UEPB). Endereço Institucional: Universidade Estadual da Paraíba, Departamento de Psicologia. Rua Baraúnas, 351. Campus Universitário. CEP 58429-500, Campina Grande - PB. E-mail: raildafernandesalves@gmail.com

Emily Souza Gaião - Psicóloga, Mestranda em Psicologia Cognitiva, Universidade Federal de Pernambuco. Endereço Institucional: Av. Prof. Moraes Rego, 1235. Cidade Universitária, Recife/PE. E-mail:emilygaiao@gmail.com

Gabriella Cézar dos Santos - Psicóloga. E-mail: gabriellacezar@ hotmail.com

Luisa de Marillac Ramos Soares - Doutora em Educação. Universidade Federal de Campina Grande. Endereço Institucional: R. Aprígio Veloso, 882. Bodocongo. CEP 58109-900 Campina Grande/PB. E-mail: luisademarillac@yahoo.com.br

Recebido em 27.04.2013

Primeira Decisão Editorial em 17.07.13

Segunda Decisão Editorial em 05.08.2013

Aceito em 11.04.2014 